\title{
A model of ideal elastomeric gels for polyelectrolyte gels
}

\section{Citation}

Li, Jianyu, Zhigang Suo, and Joost J. Vlassak. 2014. "A Model of Ideal Elastomeric Gels for Polyelectrolyte Gels." Soft Matter 10 (15): 2582. doi:10.1039/c3sm52751d.

\section{Published Version}

doi:10.1039/c3sm52751d

\section{Permanent link}

http://nrs.harvard.edu/urn-3:HUL.InstRepos:13910044

\section{Terms of Use}

This article was downloaded from Harvard University's DASH repository, and is made available under the terms and conditions applicable to Open Access Policy Articles, as set forth at http:// nrs.harvard.edu/urn-3:HUL.InstRepos:dash.current.terms-of-use\#OAP

\section{Share Your Story}

The Harvard community has made this article openly available.

Please share how this access benefits you. Submit a story.

\section{Accessibility}




\title{
Model of ideal elastomeric gels for polyelectrolyte gels
}

\author{
Jianyu Li, Zhigang Suo, Joost J. Vlassak \\ School of Engineering and Applied Sciences, Harvard University \\ 29 Oxford Street, Cambridge MA
}

Keywords

\begin{abstract}
The concept of ideal elastomeric gel is extended to polyelectrolyte gels and verified using a polyacrylamide-co-acrylic acid hydrogel as a model material system. A comparison between mixing and ion osmosis shows that the mixing osmosis is larger than the ion osmosis for small swelling ratios, while the ion osmosis dominates for large swelling ratios. We show further that the non-Gaussian chain effect becomes important in the elasticity of the polymer network at the very large swelling ratios that may occur under certain conditions of $\mathrm{pH}$ and salinity. We demonstrate that the Gent model captures the non-Gaussian chain effect well and that it provides a good description of the free energy associated with the stretching the network.
\end{abstract}

\section{INTRODUCTION}

A polyelectrolyte gel is formed when a cross-linked polymer network carrying ionizable groups, generally acidic, absorbs a solvent containing ionic species. Polyelectrolyte gels have the capacity to absorb large amounts of solvent, leading to their use in a broad range of applications in healthcare and personal hygiene. ${ }^{1}$ The degree of swelling depends sensitively on 
environmental conditions such as $\mathrm{pH}$ and salinity, ${ }^{2-4}$ and in some cases even electric fields, ${ }^{5}$ temperature, ${ }^{6}$ or light. ${ }^{7}$ Thus polyelectrolyte gels can also be used as smart materials in sensors and actuators..$^{8,9}$

These applications have motivated the development of theoretical models characterizing the chemo-mechanical behavior of polyelectrolyte gels. Katchalsky et al. pioneered the study of polyelectrolyte solutions and gels. ${ }^{10,11}$ Tanaka et al. validated the use of Donnan's theory for the osmotic pressure that develops when mixing polymer chains and ions. ${ }^{12}$ A scaling theory proposed by de Gennes et al. and further developed Rubinstein et al. was used to predict the behavior of polyelectrolyte gels as a function of chain length, type of cross-link, monomer, and salt concentrations. ${ }^{13-15}$ Several theoretical models for the swelling behavior of polyelectrolyte gels based on the Flory-Rehner theory have also been reported. ${ }^{16,17}$ Less attention has been paid to direct comparisons between experiments and theoretical predictions. A notable exception is the work by Prudnikova and Utz, ${ }^{18}$ who applied the thermodynamic field theory by Hong et al, ${ }^{19}$ to predict the Donnan potentials, but found a systematic error between theory and experiments. Evidently, a comprehensive quantitative model for the complex chemomechanical behavior of polyelectrolyte gels is still elusive.

One possible approach is to develop a model based on the concept of the ideal elastomeric gel, which was proposed recently by Cai and $\mathrm{Suo},{ }^{20}$ and verified for polyacrylamide hydrogels in an experimental study. ${ }^{21}$ This study showed that stresses applied under various loading conditions are balanced by the elasticity of the polyacrylamide network and the osmotic pressure. It was further demonstrated that the presence of cross-links does not affect the mixing energy of the polymer chains and the solvent measurably. An important feature of the ideal elastomeric gel concept is the absence of a specific statistical model for the behavior of the 
polymer network or a specific expression for the energy of mixing; a full model of a gel can then be realized through a series of simple experiments. Since the theoretical framework of the ideal elastomeric gel model is quite general, the same approach can also be applied for other types of gels.

In this paper, we apply the concept of the ideal elastomeric gel to polyelectrolyte gels using polyacrylamide-co-acrylic acid hydrogel as a model material system. We demonstrate experimentally that in polyelectrolyte gels the elasticity of the network is balanced by the osmotic pressure associated with two distinct processes: mixing of polymer and solvent, and the redistribution of the ionic species. In the present model, the contribution of the ionic species to the osmotic pressure is quantified using Donnan's theory. A comparison between the mixing osmosis and the ion osmosis shows that the mixing osmosis is larger than the ion osmosis for small swelling ratios, while the ion osmosis dominates for very large swelling ratios. We show further that the non-Gaussian chain effect becomes important in the elasticity of the polymer network at the very large swelling ratios that may occur under certain conditions of $\mathrm{pH}$ and salinity. We demonstrate that the Gent model captures the non-Gaussian chain effect well and that it provides a good description of the free energy associated with the stretching the network.

\section{THEORY}

Following our previous work, ${ }^{20,21}$ we derive the equations of state for a polyelectrolyte gel. To focus on the main ideas, we consider deformation in the principal directions, although the theory is readily expanded to the more general case. ${ }^{22}$ In the reference state, the gel is a unit cube of dry polymer, containing no solvent and subject to no applied forces (Figure 1a). In the current state, submerged in a solution and subject to applied forces, the gel absorbs solvent molecules 
and various ionic species, and stretches into a rectangular block of dimensions $\lambda_{1}, \lambda_{2}$ and $\lambda_{3}$ (Figure 1a). The volume ratio of gel to dry polymer, $J$, known as the swelling ratio, relates to the dimensions of the rectangular block by $J=\lambda_{1} \lambda_{2} \lambda_{3}$. The gel and the external solution contain four mobile species: solvent molecules, protons, counter ions with charges opposite to the charges fixed on the polymer chains, and co-ions with charges of the same sign as the fixed charges (Figure 1c). We define the nominal concentration $C_{a}$ of species $\alpha$ as the number of species $\alpha$ in the current state divided by the volume of the dry network. The same number divided by the volume of the gel in the current state defines the true concentration $c_{\alpha}$. The two quantities are evidently related by $C_{\alpha}=c_{\alpha} J$. As part of the swelling process, the acidic groups $A H$ on the polymer chains of the network may dissociate, leaving fixed charges $A^{-}$on the chains. This dissociation reaction, $A H \leftrightarrow A^{-}+H^{+}$, reaches equilibrium when

$$
\frac{C_{H^{+}} C_{A^{-}}}{C_{A H}}=\frac{N_{a} K_{a}}{J}
$$

where $N_{a}$ is Avogadro's constant and $K_{a}$ is the acid dissociation constant.

Recall the two basic assumptions underlying the concept of an ideal elastomeric gel. (1) Molecular incompressibility: the volume of the ideal elastomeric gel is equal to the sum of the volume of the dry network and that of the solvent,

$$
J=1+\Omega_{s} C_{s},
$$

where $\Omega_{s}$ is the volume per solvent molecule $s$. In the case of polyelectrolyte gels, we assume further that the concentrations of the ionic species are generally much lower than that of the solvent species,${ }^{17}$ so that Equation (2) remains valid. (2) Separability of the Helmholtz free 
energy: the Helmholtz free energy of the ideal elastomeric gel consists of one term that represents the elastic stretching of the polymer network and one term that is associated with mixing of polymer and solvent. In the case of polyelectrolyte gels, the fixed and mobile ions also contribute to the Helmholtz free energy. Following prior work, ${ }^{12,16,17}$ we formulate the total Helmholtz free energy referred to a unit volume in the reference state as the sum of the following contributions:

$$
W=W_{\text {stretch }}+W_{\text {mix }}+W_{\text {ion }}+W_{\text {dis }} \text {, }
$$

where $W_{\text {stretch }}$ is the energy contribution due to the stretching of the network. This term is a function of the stretches $\left(\lambda_{1}, \lambda_{2}, \lambda_{3}\right)$ and depends on the cross-link density of the network. The term $W_{m i x}$ is the contribution associated with the mixing of polymer and solvent. This term is a function of the swelling ratio, $J$, given by Eq. (2) and is taken independent of the cross-link density. The term $W_{\text {ion }}$ is associated with the mixing of mobile ions and polymer and generally takes the form $W_{i o n}=W_{i o n}\left(J, C_{H^{+}}, C_{+}, C_{-}\right)$, where the subscripts + and - refer to the counter ions and co-ions, respectively. If the concentrations of the ions are low and the polymer network is weakly charged, the electrostatic interactions between the ions in the gel is negligible ${ }^{12}$ and $W_{\text {ion }}$ is determined mainly by the entropy of mixing of the mobile ions and the polymer network:

$$
W_{\text {ion }}=k T\left[C_{H^{+}}\left(\log \frac{C_{H^{+}}}{c_{H^{+}}^{r e f} J}-1\right)+C_{+}\left(\log \frac{C_{+}}{c_{+}^{r e f} J}-1\right)+C_{-}\left(\log \frac{C_{-}}{c_{-}^{r e f} J}-1\right)\right],
$$

where $c^{\text {ref }}$ refers to the standard concentration, defined as $1 \mathrm{~mol} / \mathrm{L}$. For strongly charged polyelectrolyte gels, the energy contribution due to electrostatic interactions between mobile ions and fixed charges may be significant and needs to be considered. ${ }^{11,23}$ The electrostatic effect is 
readily added into the theoretical framework, but is not further considered in this study. The last term in Eq. (3), $W_{\text {dis }}$, denotes the change in free energy caused by dissociation of the acidic groups on the polymer chains. This term contains both an entropic and an enthalpic term,

$$
W_{d i s}=k T\left[C_{A^{-}} \log \left(\frac{C_{A^{-}}}{C_{A^{-}}+C_{A H}}\right)+C_{A H} \log \left(\frac{C_{A H}}{C_{A^{-}}+C_{A H}}\right)\right]+\gamma C_{A^{-}},
$$

where $\gamma$ is the increase in enthalpy when an acidic group dissociates. Under the constraints of mass conservation and electroneutrality, $C_{A^{-}}$and $C_{A H}$ are readily expressed in terms of $C_{H^{+}}$, $C_{+}$and $C_{-}$:

$$
C_{A^{-}}=C_{H^{+}}+C_{+}-C_{-}
$$

and

$$
C_{A H}=f / v-\left(C_{H^{+}}+C_{+}-C_{-}\right)
$$

where $f$ is the number of the acidic groups attached to the network divided by the total number of monomers forming the network, and $v$ is the volume per monomer. Consequently, the total Helmholtz free energy can be written as a function of six independent variables: the stretches $\left(\lambda_{1}, \lambda_{2}, \lambda_{3}\right)$ and the concentrations of the protons, counter ions, and co-ions $\left(C_{H^{+}}, C_{-}, C_{+}\right)$.

When a dry polymer network is submerged in a solution and subjected to forces, the network absorbs the mobile species and deforms, eventually attaining a state of thermodynamic equilibrium. The condition for equilibrium can be formulated as follows. Define the stresses $\sigma_{1}$, $\sigma_{2}$ and $\sigma_{3}$ as the applied forces divided by the areas of the faces of the block of gel in the current state (Figure 1a). Thus, the forces on the faces of the rectangular block are $\sigma_{1} \lambda_{2} \lambda_{3}$, $\sigma_{2} \lambda_{3} \lambda_{1}$ and $\sigma_{3} \lambda_{1} \lambda_{2}$. Associated with a small change in the dimensions of the gel, these forces 
perform work on the gel $\sigma_{1} \lambda_{2} \lambda_{3} d \lambda_{1}+\sigma_{2} \lambda_{3} \lambda_{1} d \lambda_{2}+\sigma_{3} \lambda_{1} \lambda_{2} d \lambda_{3}$. The gel and the solution can also exchange four mobile species: solvent molecules, protons, counterions and co-ions. Let $\mu_{\alpha}$ be the chemical potential of species $\alpha-$ since the gel and the solution are in a state of equilibrium, the chemical potential of each species is uniform in the system. When a number $d C_{a}$ (per unit volume of dry gel) of species $\alpha$ are transferred from the solution to the gel, the free energy of the gel changes by $\mu_{\alpha} d C_{\alpha}$. This statement holds for solvent molecules, counter ions, and co-ions but not for protons because the number of protons in the gel can also change by dissociation of the acidic groups on the polymer chains. Consequently, in equilibrium the total Helmholtz free energy $W$ obeys

$$
\begin{aligned}
& d W=\sigma_{1} \lambda_{2} \lambda_{3} d \lambda_{1}+\sigma_{2} \lambda_{3} \lambda_{1} d \lambda_{2}+\sigma_{3} \lambda_{1} \lambda_{2} d \lambda_{3} \\
& +\mu_{s} d C_{s}+\mu_{H^{+}}\left(d C_{H^{+}}-d C_{A^{-}}\right)+\mu_{-} d C_{-}+\mu_{+} d C_{+} .
\end{aligned}
$$

Combining Eqs. (2)-(8) yields the following expression,

$$
\begin{aligned}
& {\left[\frac{\partial W_{\text {stretch }}}{\partial \lambda_{1}}-\left(\sigma_{1}+\Pi_{\text {mix }}+\Pi_{\text {ion }}\right) \lambda_{2} \lambda_{3}\right] d \lambda_{1}} \\
& +\left[\frac{\partial W_{\text {stretch }}}{\partial \lambda_{2}}-\left(\sigma_{2}+\Pi_{\text {mix }}+\Pi_{\text {ion }}\right) \lambda_{3} \lambda_{1}\right] d \lambda_{2} \\
& +\left[\frac{\partial W_{\text {stretch }}}{\partial \lambda_{3}}-\left(\sigma_{3}+\Pi_{\text {mix }}+\Pi_{\text {ion }}\right) \lambda_{1} \lambda_{2}\right] d \lambda_{3} \\
& +\left[\frac{\partial W}{\partial C_{+}}-\left(\mu_{+}-\mu_{H^{+}}\right)\right] d C_{+}+\left[\frac{\partial W}{\partial C_{-}}-\left(\mu_{-}+\mu_{H^{+}}\right)\right] d C_{-} \\
& +\frac{\partial W}{\partial C_{H^{+}}} d C_{H^{+}}=0
\end{aligned}
$$

where

$$
\Pi_{m i x}=-\frac{d W_{m i x}(J)}{d J},
$$




$$
\Pi_{i o n}=-\frac{\partial W_{i o n}\left(J, C_{H^{+}}, C_{+}, C_{-}\right)}{\partial J}+\frac{\mu_{s}}{\Omega_{s}} .
$$

Here $\Pi_{m i x}$ is the osmotic pressure in the gel in equilibrium with the pure solvent, while $\Pi_{i o n}$ represents the osmotic pressure in the gel due to the mobile ions; $\mu_{s} / \Omega_{s}$ is the osmotic pressure in the gel due to the chemical potential of the solvent in the external solution containing ionic species:

$$
\mu_{s}=-k T \Omega_{S}\left(c_{H^{+}}^{*}+c_{+}^{*}+c_{-}^{*}\right) .
$$

where $c_{\alpha}^{*}$ is the true concentration of species $\alpha$ in the external solution. ${ }^{17}$ Substituting Eqs. (4) and (12) into (11) yields

$$
\Pi_{i o n}=k T\left(c_{H^{+}}+c_{+}+c_{-}-c_{H^{+}}^{*} c_{+}^{*}-c_{-}^{*}\right) .
$$

The network, the solvent and the applied forces are in equilibrium, if Eq. (9) holds for arbitrary and independent small changes in the three stretches $\left(\lambda_{1}, \lambda_{2}\right.$ and $\left.\lambda_{3}\right)$ and the three nominal concentrations $\left(C_{+}, C_{-}, C_{H^{+}}\right)$. Consequently, the expressions in the brackets in front of each of the six terms in Eq. (9) must vanish individually, yielding the following equations:

$$
\begin{gathered}
\sigma_{1}=\frac{\partial W_{\text {stretch }}}{\lambda_{2} \lambda_{3} \partial \lambda_{1}}-\Pi_{m i x}-\Pi_{i o n}, \\
\sigma_{2}=\frac{\partial W_{\text {strecth }}}{\lambda_{1} \lambda_{3} \partial \lambda_{2}}-\Pi_{m i x}-\Pi_{i o n}, \\
\sigma_{3}=\frac{\partial W_{\text {stretch }}}{\lambda_{1} \lambda_{2} \partial \lambda_{3}}-\Pi_{m i x}-\Pi_{i o n} . \\
\frac{\partial W}{\partial C_{+}}=\mu_{+}-\mu_{-} .
\end{gathered}
$$




$$
\begin{gathered}
\frac{\partial W}{\partial C_{-}}=\mu_{-}+\mu_{H^{+}} . \\
\frac{\partial W}{\partial C_{H^{+}}}=0 .
\end{gathered}
$$

The applied stresses are balanced by the elasticity of the network, the osmotic pressure due to mixing of polymer and solvent, and the osmotic pressure due to mixing of polymer and ions. Equations (14) express mechanical equilibrium; Equations (15) provide the equilibrium conditions with respect to the exchange of counter ions and co-ions between the gel and its surroundings; Equation (16) formulates the equilibrium condition for dissociation of the acidic groups on the polymer chains. Substituting Eqs (3)-(5) into (16) leads to

$$
N_{A} K_{a}=c_{H^{+}}^{r e f} \exp \left(-\frac{\gamma}{k T}\right)
$$

Equations (2), (14), (15), and (16) constitute the equations of state of the polyelectrolyte gel, relating the thermodynamic variables: $\lambda_{1}, \lambda_{2}, \lambda_{3}, \sigma_{1}, \sigma_{2}, \sigma_{3}, C_{+}, C_{-}, C_{H^{+}}$. Once the free energy contribution due to stretching of the network, $W_{\text {stretch }}$, and the osmotic pressure, $\Pi_{m i x}$, have been characterized experimentally, the equations of state provide a complete description of the chemomechanical behavior of the polyelectrolyte gel. The energy contribution due to stretching is readily determined from stress-strain measurements. Characterizing $\Pi_{i o n}$ is less straightforward since that requires knowledge of the concentrations $\left(C_{H^{+}}, C_{+}, C_{-}\right)$inside the gel, which are difficult to measure in practice. ${ }^{24}$ Instead, we adopt Donnan's theory ${ }^{17}$, which provides a relationship between the ion concentrations inside the gel and those in the external solution,

$$
\frac{c_{+}}{c_{+}^{*}}=\frac{c_{H^{+}}}{c_{H^{+}}^{*}}=\left(\frac{c_{-}}{c_{-}^{*}}\right)^{-1} .
$$


Tanaka et al. successfully applied Donnan's theory to capture the swelling behavior of an ionic gel quantitatively. ${ }^{12}$ The concentrations of the three species inside the gel also satisfy the equilibrium condition (1) for dissociation of the acid groups, yielding

$$
\frac{c_{H^{+}}\left(c_{H^{+}}+c_{+}-c_{-}\right)}{\frac{f}{v J}-\left(c_{H^{+}}+c_{+}-c_{-}\right)}=N_{A} K_{a} .
$$

If $f, J, c_{H^{+}}^{*}, c_{+}^{*}, c_{-}^{*}$, and $K_{a}$ are known, the concentrations $\left(c_{H^{+}}, c_{+}, c_{-}\right)$of the ionic species in the gel are solved readily from Eqs. (18) and (19). 


\section{EXPERIMENTAL SECTION}

To determine the free energy contributions of stretching and the osmotic pressure in the gels in equilibrium with the pure solvent, and to verify the equations of state for polyelectrolyte gels, a series of polyacrylamide-co-acrylic acid gels were synthesized according to the following protocol; ${ }^{12}$ a summary of the composition and designation of each sample is provided in Table 1. Acrylamide (AAM), acrylic acid (AA), N,N'-methylenebis(acrylamide) (MBAA) and ammonium persulfate (APS) were acquired from Sigma Aldrich. The overall monomer $(\mathrm{AAM}+\mathrm{AA})$ concentration was $1000 \mathrm{mM}$. As indicated in Table 1 , the weight fraction of acrylic acid, $f$, was varied systematically, but kept small to ensure a low density of acidic groups on the polymer chains. Different cross-link densities were obtained by varying the weight percentage of MBAA (Table 1). APS (7 mM) was added as initiator for the free-radical polymerization. The chemicals (AAM, AA, MBAA and APS) were mixed with $10 \mathrm{~mL}$ syringes. Part of the mixture $(6 \mathrm{~mL})$ was poured into a glass mold of $1 \mathrm{~mm}$ thickness. The rest of the solution $(4 \mathrm{~mL})$ was kept in the syringe to fabricate samples with a cylindrical shape.

Gelation occurred over a period of one hour on a hot plate at $70^{\circ} \mathrm{C}$. To prevent hydrolysis of acrylamide and to precisely control the fraction of acidic groups, we did not use the usual accelerator, tetramethyl-ethylenediamine (TEMED). After gelation, the samples were stored at room temperature for 24 hours, and then transferred to a large body of distilled water to remove unreactive chemicals and other impurities, while the gels were allowed to swell freely to equilibrium. This step lasted around 10 days, during which fresh distilled water was added every 24 hours. 
The masses of the fully swollen cylindrical samples $\left(m_{g e l}\right)$ were measured using an analytical scale with an accuracy of $10 \mu \mathrm{g}$. The samples were subsequently were frozen at a temperature of $-80^{\circ} \mathrm{C}$ and transferred to a freeze-dry system (Labconco Corporation) with a collector temperature of $-50^{\circ} \mathrm{C}$ and a vapor pressure of 0.040 mbar. This dehydration process took three days to complete, after which the mass of the dehydrated samples $\left(m_{d r y}\right)$ was measured. Swelling ratios were then calculated using

$$
J=1+\frac{\left(m_{g e l}-m_{d r y}\right) / \rho_{\text {water }}}{m_{d r y} / \rho_{d r y}}
$$

where $\rho_{\text {water }}$ is the density of water taken as $1.000 \mathrm{~g} / \mathrm{cm}^{3}$, and $\rho_{\text {dry }}$ is the density of the dry polymer network taken as the density of polyacrylamide at $1.443 \mathrm{~g} / \mathrm{cm}^{3}{ }^{25}$ The swelling ratios, $J_{o}$, in distilled water are listed in Table 1 for each of the samples. Swelling ratios in subsequent experiments were calculated from $J_{o}$ and the change in diameter, $J=J_{o}\left(D_{f} / D_{i}\right)^{3}$, where $D_{i}$ and $D_{f}$ are the initial and final diameter of the sample.

The responses of the gels to changes in $\mathrm{pH}$ and salinity of the external solution were characterized using samples AA04-080 and AA04-433. Small gel cylinders of diameters 8 or 12 $\mathrm{mm}$ were punched out of fully swollen gel sheets. These samples were transferred to solutions of $\mathrm{pH}$ varying from 2.5 to 12 . Acidic solutions $(\mathrm{pH}<7)$ were prepared by adding $\mathrm{HCl}$ to distilled water, while $\mathrm{KOH}$ was used for alkaline solutions $(\mathrm{pH}>7)$. Gel cylinders were also transferred to solutions with $\mathrm{NaCl}$ concentrations varying from $10^{-7} \mathrm{M}$ to $10^{-2} \mathrm{M}$. The use of buffer solutions was avoided to not introduce additional electrochemically active species. The external solutions were renewed every $24 \mathrm{hrs}$. We recorded the diameters of the cylinders using a pair of calipers before and after reaching the new equilibrium state. 
Small gel cylinders with $8 \mathrm{~mm}$ diameters were used for the mechanical tests. A series of uniaxial compression tests were performed using an AR-G2 rheometer (TA Instruments). The rheometer platen approached the samples at a speed of $5 \mu \mathrm{m} / \mathrm{s}$ until a rise in force was detected, indicating contact between the platen and the gel. Upon the contact, the displacement rate of the platen was increased to $20 \mu \mathrm{m} / \mathrm{s}$ until a pre-determined stretch ratio, typically $0.8-0.9$, was achieved. The entire loading process was finished within 2 minutes to avoid any redistribution of solvent inside the gel and thus maintain incompressibility. Both force and displacement were recorded continuously throughout the experiment. 


\section{RESULTS AND DISCUSSION}

\section{Mechanical characterization}

Figure 2a shows the swelling ratios in distilled water for each of the samples. Typical values are well in excess 50 so that the water content of most swollen gels is greater than $95 \%$. As illustrated in the figure, the swelling ratio changes rapidly with the fraction of acidic groups and the cross-link density. Even in distilled water, weakly charged and loosely cross-linked gels (e.g. AA02-080 and AA04-080) can swell to more than 800 times their dry volume.

The contribution of the free energy of stretching, $W_{\text {stretch }}$, was determined from uniaxial compression tests on samples fully swollen in distilled water. During the tests, the gels behaved like incompressible solids $\left(\lambda_{1} \lambda_{2} \lambda_{3}=\lambda_{o}^{3}\right)$, because there was insufficient time for diffusion. We then used the Gaussian chain model,

$$
\begin{gathered}
\sigma_{3}-\sigma_{1}=\frac{N k T}{\lambda_{\mathrm{o}}}\left[\left(\frac{\lambda_{3}}{\lambda_{\mathrm{o}}}\right)^{2}-\left(\frac{\lambda_{1}}{\lambda_{\mathrm{o}}}\right)^{2}\right], \\
\sigma_{3}-\sigma_{2}=\frac{N k T}{\lambda_{\mathrm{o}}}\left[\left(\frac{\lambda_{3}}{\lambda_{\mathrm{o}}}\right)^{2}-\left(\frac{\lambda_{2}}{\lambda_{\mathrm{o}}}\right)^{2}\right],
\end{gathered}
$$

to fit the experimental stress-stretch relations, where $\lambda_{o}=J_{o}^{1 / 3}$ is the stretch ratio after swelling in distilled water, $N$ is the number of polymer chains per unit volume of the dry network, $k$ is Boltzmann's constant, and $T$ is the absolute temperature. Considering uniaxial compression along the $x_{3}$-direction, these equations reduce to a single equation

$$
\sigma_{3}=\frac{N k T}{\lambda_{o}}\left(\lambda^{2}-\frac{1}{\lambda}\right)
$$


where $\lambda=\lambda_{3} / \lambda_{0}$ is the stretch relative to the freely swollen state. It is evident from Equation (22) that $N k T / \lambda_{\text {o }}$ represents Young's modulus of the gels, while also allowing us to determine the cross-link density $(N)$ from the initial slope of the stress-stretch curve. In our previous work on polyacrylamide gels, the Gaussian-chain model provided an excellent description of the mechanical behavior of the gels. As expected, the Gaussian-chain model also provides a very good fit for the experimental stress-stretch curves for the polyacrylamide-co-acrylic acid gels.

Figure 2(b) illustrates that $N k T$ increases with increased cross-linker, but is independent of the fraction of acrylic acid, $f$. A correlation between the modulus and the ionic components inside the gel has been reported in the literature, ${ }^{13}$ a phenomenon attributed to the electrostatic interaction between the fixed ions on the polymer chain. In this study, the fraction of acidic group is intentionally maintained at a level below 5\%, so that fixed ion are on average separated by at least twenty neutral segments resulting in weak electrostatic interaction between the ions.

\section{Characterization of the osmotic pressure terms}

The osmotic pressure $\Pi_{i o n}$ of the gel samples can be calculated by combining Equation (13) with Equations (18) and (19). The results for an external solution of distilled water with a slightly acidic $\mathrm{pH}$ of 6.5 are plotted as a function of $f / J$ in Figure $3 \mathrm{a}$ - data for gels with different cross-link density and acid group content are shown, but they collapse into a single curve because the ionic osmotic pressure is a unique function of the current concentration of acid groups, $f / J$, and the $\mathrm{pH}$ of the external solution. The ionic osmotic pressure is shown to increase with acid group content and decrease with decreasing cross-link density. The former is caused by the higher concentration of active species, the latter occurs because the equilibrium swelling ratio is larger, which results in a lower concentration of active species. 
Using the equations of state, it is now possible to derive the function $\Pi_{m i x}(J)$ from our experiments without assuming a specific model for $W_{\text {mix }}$. The stress-strain curves suggest that $W_{\text {stretch }}$ is well described by the Gaussian-chain model, i.e.,

$$
W_{\text {strecth }}=\frac{1}{2} N k T\left[\lambda_{1}^{2}+\lambda_{2}^{2}+\lambda_{3}^{2}-3-2 \log \left(\lambda_{1} \lambda_{2} \lambda_{3}\right)\right] .
$$

Under free-swelling conditions, the stretches are isotropic, $\lambda_{1}=\lambda_{2}=\lambda_{3}=J_{o}^{1 / 3}$ and all applied stress components vanish, $\sigma_{1}=\sigma_{2}=\sigma_{3}=0$. Substituting into Eq. (14) yields

$$
\Pi_{m i x}=\frac{N k T}{J_{o}}\left(J_{o}^{\frac{2}{3}}-1\right)-\Pi_{i o n},
$$

from which $\Pi_{\text {mix }}$ can be obtained directly once $J_{o}, N k T$, and $\Pi_{i o n}$ have been determined experimentally. Figure $3(\mathrm{~b})$ plots the function, $\Pi_{\operatorname{mix}}$, as a function of swelling ratio for gels with different cross-link densities and acid contents. We have also included $\Pi_{\operatorname{mix}}$ measured for polyacrylamide (PAAM) hydrogels in our previous work. Regardless of hydrogel cross-link density or acid content, $\Pi_{m i x}$ collapses into a single master curve over a large range of swelling ratios, i.e., $\Pi_{m i x}$ is a function of a single variable, $J$. This is a direct consequence of the low concentration of acid groups in the hydrogels, which makes the overall chemistry of the polymer chains close to that of pure polyacrylamide.

The responses of the gels to changes in $\mathrm{pH}$ or salinity of the external solution were also characterized. Figure 4 (a) plots the swelling ratio as a function of $\mathrm{pH}$, along with experimental data obtained by Tanaka et al. ${ }^{12}$ A qualitative understanding of the trends in the data is as follows. When the $\mathrm{pH}$ of the external solution is low, protons are abundant and the acidic groups on the polymer chains are fully protonated. The gel behaves like a neutral gel with a relatively 
small swelling ratio because of the absence of an ionic osmotic pressure $\Pi_{i o n}$. As the $\mathrm{pH}$ approaches 8, the acidic groups dissociate and create fixed charges on the polymer chains. An imbalance of mobile ions builds up between the gel and the external solution, the increasing $\Pi_{i o n}$ , makes the gel swell. Once the $\mathrm{pH}$ increases further, the acidic groups are fully dissociated and the ionic osmotic pressure $\Pi_{i o n}$ drops because of the abundant presence of mobile ions everywhere. The response to the changes in salinity, the concentration of $\mathrm{NaCl}$ in the external solution, is shown in Figure 4(b); an understanding similar to that for Figure 4(a) applies here. The gel acts as an ion exchanger. As the concentration of $\mathrm{NaCl}$ increases, protons in the gel are exchanged with $\mathrm{Na}^{+}$ions and the acidic groups continue to dissociate. At approximately $10^{-4} \mathrm{M}$ of $\mathrm{NaCl}$, the acidic groups are completely dissociated. The excess of $\mathrm{NaCl}$ reduces the ion imbalance between the gel and the solution, and the degree of swelling decreases. In the model we assumed an ideal ionic solution and insignificant effects due to electrostatic interactions between fixed and mobile ions, both valid for low concentrations $\left(<10^{-2} \mathrm{M}\right)$ of ions in the external solution. For strong electrolyte solutions, the Debye-Huckel model may need to be invoked to calculate the activity of the ions.

With known cross-link density, swelling ratio, and concentrations of ion species in the external solution $\left(c_{H^{+}}^{*}, c_{+}^{*}, c_{-}^{*}\right)$, the ionic osmotic pressure $\Pi_{i o n}$ can be calculated from the model. We also used the model to re-analyze data from Tanaka et al. ${ }^{12}$ Based on the concentration of MBAA in their gels, we estimated that $N k T \sim 9 \mathrm{kPa}$ for their gels. Figures $5(\mathrm{a})$ and (b) plot the ionic osmotic pressure versus equilibrium swelling ratio for external solutions of various $\mathrm{pH}$ and $\mathrm{NaCl}$ concentration, respectively. Results are shown for two hydrogels with identical acid content but different cross-link densities, along with the re-analyzed results from Tanaka et al. ${ }^{12}$ Once the equilibrium swelling ratio and the ionic osmotic pressure have been determined, the 
osmotic pressure due to mixing can be calculated from Eq. (24). The results are illustrated in Figures 6(a) and (b). It is evident that the osmotic pressure data for PAAM and AA04-080 form a single master curve that decays with swelling ratio. For gel AA04-433 and for Tanaka's gel, however, we observe a discrepancy: the osmotic pressure functions drop below the master curve at large swelling ratios. We attribute this discrepancy to the use of the Gaussian chain model to describe the mechanical behavior of the network for the very large swelling ratios attained in Figures 6(a) and (b). At small swelling ratios, the polymer strands between two cross-links behave like Gaussian chains. At large swelling ratios, however, the polymer strands are highly extended in all three primary directions approaching their contour length; the network stiffens and no longer follows Gaussian elasticity. This stiffening effect is well known and has been characterized experimentally by measuring the shear modulus as a function of swelling ratio. ${ }^{14,26}$ Consequently, using Equation (24) in the model is no longer appropriate and results in erroneous values for the osmotic pressure of mixing at large swelling ratios.

\section{Use of the Gent model}

To account for the non-Gaussian chain effect, many constitutive models have been proposed..$^{27-29}$ Among these models, the model by $\mathrm{Gent}^{29}$ is an empirical two-constant constitutive relation that is essentially equivalent to the 8 -chain model $^{27}$ without involving inverse Langevin relations. The compressible form of the Gent model yields the following expression for the stretching energy

$$
W_{\text {stretch }}=-\frac{1}{2} N k T\left[J_{\lim } \log \left(1-\frac{J_{1}}{J_{\lim }}\right)+2 \log (J)\right]
$$

where $J_{1}$ is the first stretch invariant, defined by $J_{1}=\lambda_{1}^{2}+\lambda_{2}^{2}+\lambda_{3}^{2}-3$, and $J_{\text {lim }}$ denotes the maximum value of $J_{1} 3^{30}$ At small strains, Eq. (25) reduces to the Gaussian chain model Eq.(23), 
but as $J_{1}$ approaches $J_{l i m}$, the model captures the limited extensibility of the network. Due to its simplicity and robustness, the Gent model has been applied to a broad range of material systems. ${ }^{28,31,32}$ Substituting Eq. (25) into Eq. (14), we obtain the following equations of state:

$$
\begin{aligned}
& \sigma_{1}=\frac{N k T}{J}\left[\frac{J_{\lim } \lambda_{1}^{2}}{J_{\lim }-\left(\lambda_{1}^{2}+\lambda_{2}^{2}+\lambda_{3}^{2}-3\right)}-1\right]-\left(\Pi_{m i x}+\Pi_{i o n}\right) \\
& \sigma_{2}=\frac{N k T}{J}\left[\frac{J_{\lim } \lambda_{2}^{2}}{J_{\lim }-\left(\lambda_{1}^{2}+\lambda_{2}^{2}+\lambda_{3}^{2}-3\right)}-1\right]-\left(\Pi_{m i x}+\Pi_{i o n}\right) \\
& \sigma_{3}=\frac{N k T}{J}\left[\frac{J_{\lim } \lambda_{3}^{2}}{J_{\lim }-\left(\lambda_{1}^{2}+\lambda_{2}^{2}+\lambda_{3}^{2}-3\right)}-1\right]-\left(\Pi_{m i x}+\Pi_{i o n}\right)
\end{aligned}
$$

For free-swelling experiments $\left(\sigma_{1}=\sigma_{2}=\sigma_{3}=0\right.$ and $\left.\lambda_{1}=\lambda_{2}=\lambda_{3}=\lambda_{o}\right)$, we find the following expression for the osmotic pressure of mixing

$$
\Pi_{m i x}=\frac{N k T}{J}\left(\frac{J_{\lim } \lambda_{o}^{2}}{J_{\lim }-3 \lambda_{o}^{2}+3}-1\right)-\Pi_{i o n}
$$

We determine the values of $J_{\text {lim }}$ for AA04-433 and for Tanaka's gel by fitting the osmotic pressure given by Eq. (27) to the master curved obtained for PAAM. As illustrated in Figure 7, good agreement is obtained between the PAAM data and the osmotic pressure based on the Gent model with $J_{l i m}$-values of 260 and 580 for AA04-433 and for Tanaka's gel, respectively. While the Gent model is empirical and it is difficult to assign an exact quantitative interpretation to $J_{\text {lim }}$, it is possible to understand this result qualitatively: in a loosely cross-linked gel, the contour lengths of the polymer strands between cross-links are much larger than in a highly cross-linked gel, and the corresponding extensibility limit occurs at much larger strain. Thus the results are consistent with the fact that Tanaka's gels are more loosely cross-linked than AA04-433. 


\section{CONCLUSION}

In this paper we have developed the concept of ideal elastomeric gel for polyelectrolyte gels and use polyacrylamide-co-acrylic acid hydrogel as a model material system to characterize the swelling behavior as a function of the cross-link density, $\mathrm{pH}$ and salinity. In this model, stresses applied to a polyelectrolyte gel are balanced by the elasticity of the network, the osmosis pressure due to mixing of polymer and solvent, and the osmosis pressure due to ion distribution. The ion osmotic pressure is quantified using Donnan's theory, while the mixing osmosis is determined experimentally to yield a master curve that is valid for a broad range of polyacrylamide-co-acrylic acid hydrogels. Because polyelectrolyte gels can attain very large swelling ratios, non-Gaussian chain effects may need to be accounted for in the description of the mechanical behavior of the polymer network. These effects are well described by the Gent model. 
Table 1. Composition and parameters of polyacrylamide-co-acrylic acid gels. $f$ is the fraction of AA versus AAM, $J_{o}$ is the equilibrium swelling ratio of the gel in distilled water, $N k T$ is the crosslink density.

\begin{tabular}{|c|c|c|c|c|}
\hline Notation & MBAA (wt\%) & $\bar{f}$ & $J_{o}$ & $N k T(\mathrm{kPa})$ \\
\hline AA01-080 & 0.80 & 0.01 & 140 & 2.33 \\
\hline AA02-080 & 0.80 & 0.02 & 590 & 3.62 \\
\hline AA04-080 & 0.80 & 0.04 & 654 & 3.71 \\
\hline AA01-216 & 2.16 & 0.01 & 71 & 10.83 \\
\hline AA02-216 & 2.16 & 0.02 & 79 & 10.22 \\
\hline AA04-216 & 2.16 & 0.04 & 88 & 10.07 \\
\hline AA01-433 & 4.33 & 0.01 & 36 & 15.96 \\
\hline AA02-433 & 4.33 & 0.02 & 37 & 22.22 \\
\hline AAO4-433 & 4.33 & 0.04 & 61 & 17.39 \\
\hline
\end{tabular}




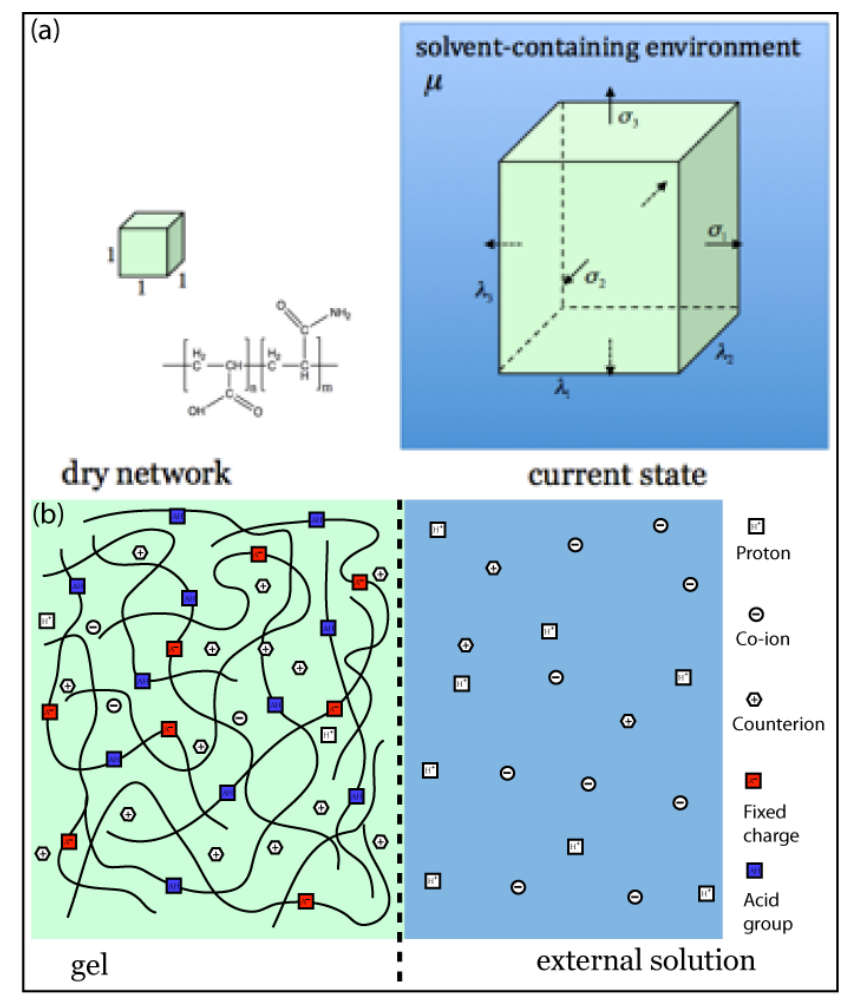

Figure 1. (a) A unit block of polyelectrolyte gel is submerged in a solution environment containing various ionic species. The inset is the structural formula of polyacrylamide-co-acrylic acid. (b) The polyelectrolyte gel, composed of cross-linked polymer chains with fixed charges and acidic groups, absorbs solvent and mobile ions, while the external solution contains solvent, protons, co-ions and counterions. 
(a)

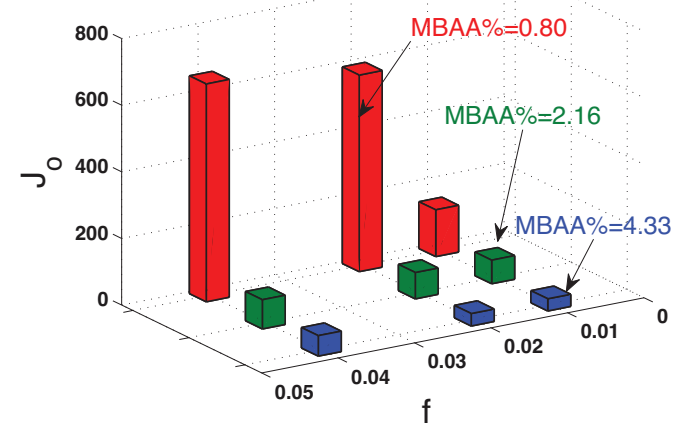

(b)

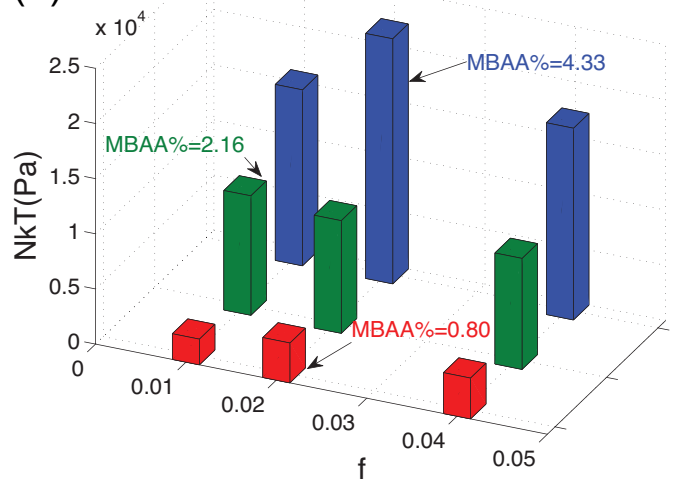

Figure 2. (a) The equilibrium swelling ratio of the gels fully swollen in distilled water $\left(J_{o}\right)$ and (b) the crosslink density ( $N k T)$ of the gels, obtained from the stress-stretch measurements, is plotted versus the fraction of acidic groups $(f)$ and the crosslinker input (MBAA\%). 
(a)

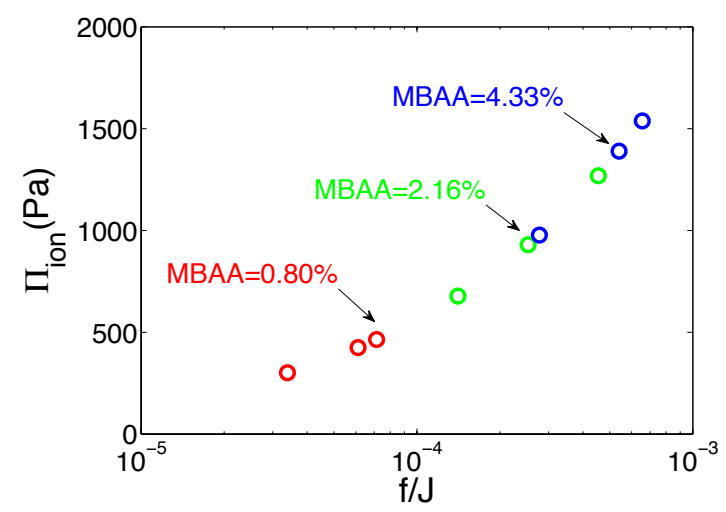

(b)

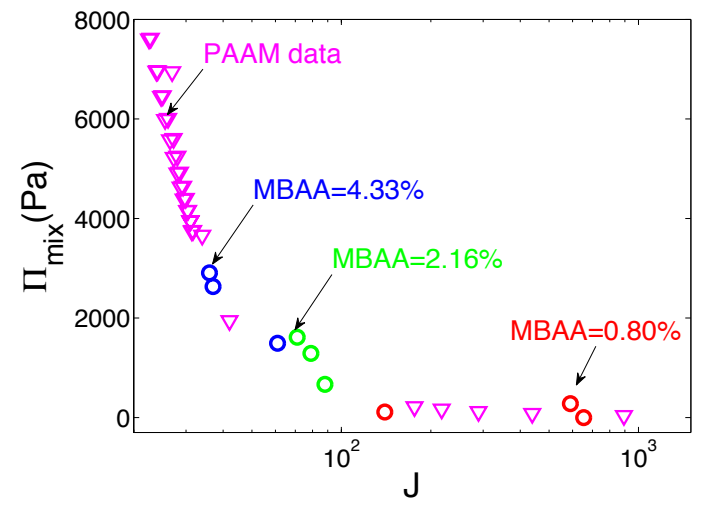

Figure 3. (a) The calculated osmotic pressure due to ions $\left(\Pi_{i o n}\right)$ versus the density of acidic groups $(f / J)$, and (b) the osmotic pressure due to mixing $\left(\Pi_{m i x}\right)$ as a function of the swelling ratio $(J)$, measured from gels fully swollen in distilled water. The same color implies the same input of crosslinker (MBAA) in the synthesis, while the fraction of acidic groups $(f)$ is varied. The data points marked by PAAM data are extracted from the measurement on polyacrylamide gels. ${ }^{20}$ 
(a)

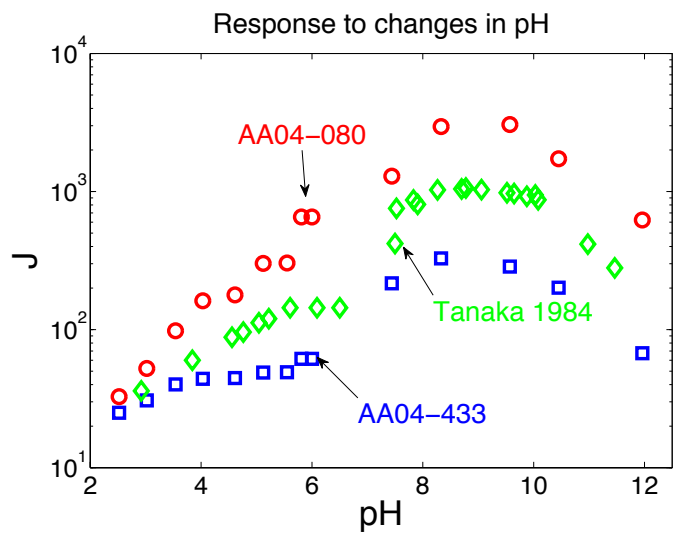

(b)

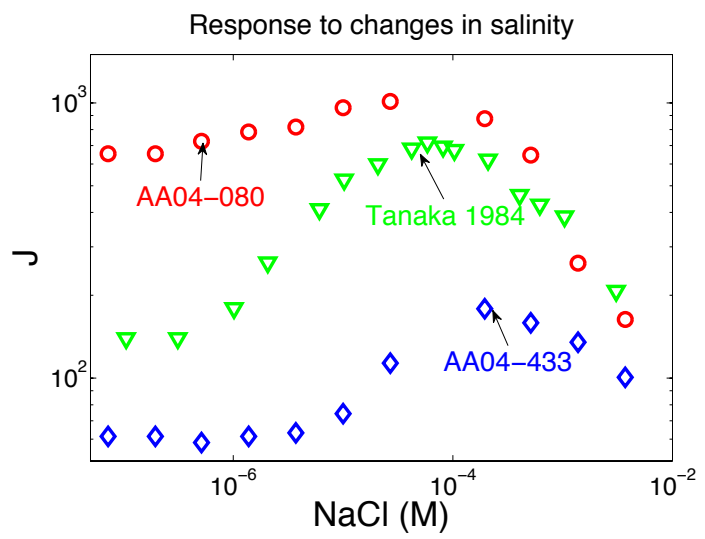

Figure 4. (a) Swelling ratio $(J)$ of the gels as a function of $\mathrm{pH}$ in the external solution; $\mathrm{HCl}$ is added for $\mathrm{pH}$ values below 7 , while $\mathrm{KOH}$ for $\mathrm{pH}$ values above 7. (b) Swelling ratio $(J)$ of the gels as a function of salt concentration $(\mathrm{NaCl})$ in the external solution. The data marked by Tanaka 1984 were obtained from an analysis of the results by Tanaka et al. 
(a)

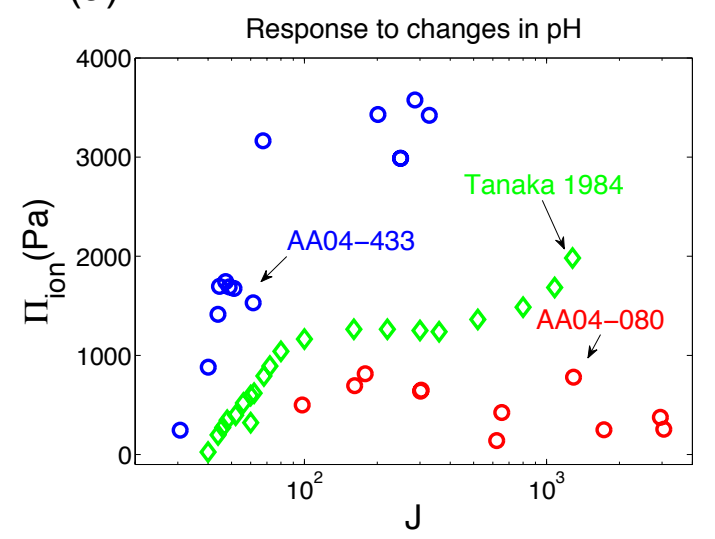

(b)

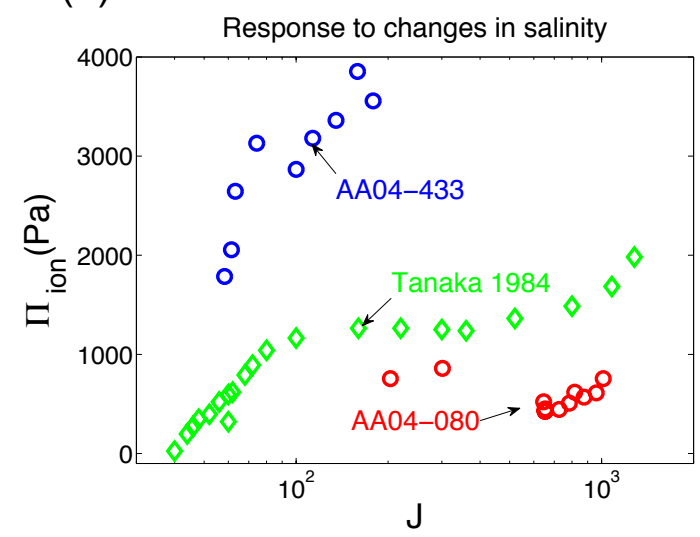

Figure 5. The osmotic pressure due to ions $\left(\Pi_{i o n}\right)$ as a function of the swelling ratio, determined from gels that were allowed to swell freely in an external solution of different $\mathrm{pH}$ values (a) and of different $\mathrm{NaCl}$ concentrations (b). 
(a)

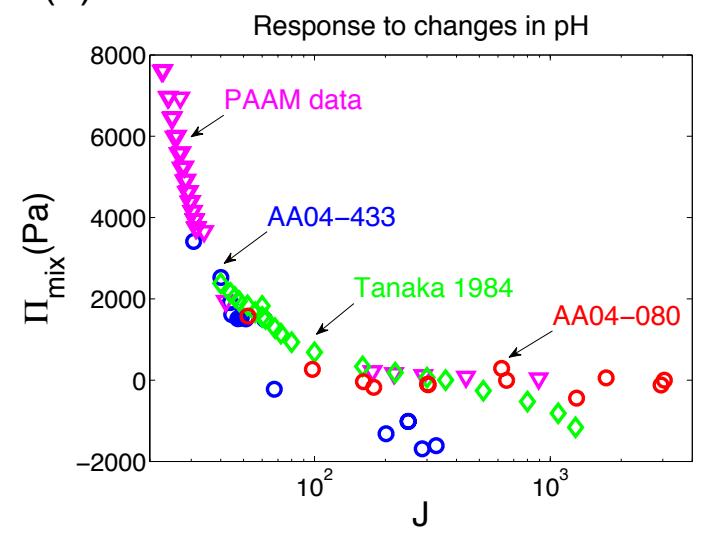

(b)

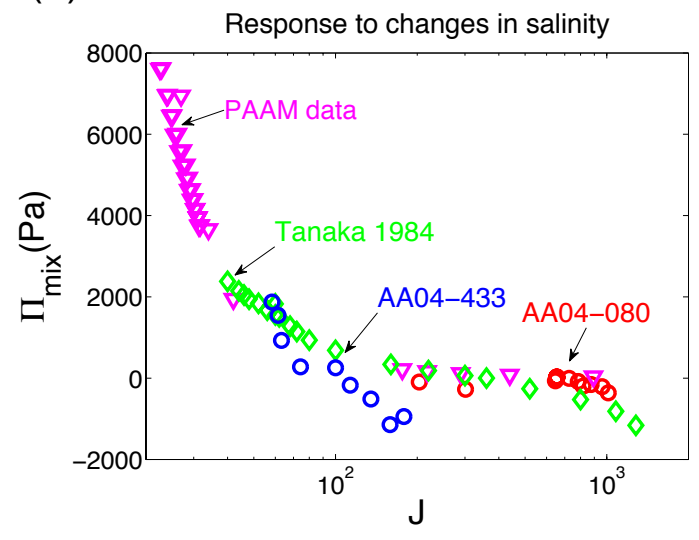

Figure 6. The osmotic pressure due to mixing $\left(\Pi_{m i x}\right)$ versus swelling ratio, determined from gels that were allowed to swell freely in an external solution of different $\mathrm{pH}$ values (a) and of different $\mathrm{NaCl}$ concentrations (b). The results were calculated from Eq. (24) using the Gaussian chain model for $W_{\text {stretch. }}$ 


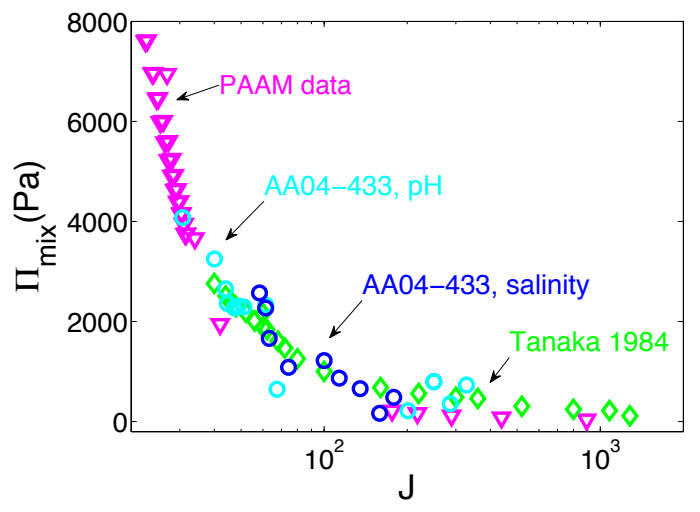

Figure 7. The osmotic pressure due to mixing $\left(\Pi_{m i x}\right)$ fitted by the equation based on Gent model, with fitting parameter $J_{\text {lim }} \approx 260$ for AA04-433 and $J_{\text {lim }}=580$ for Tanaka's gel. 


\section{REFERENCE}

(1) Dubrovskii, S. A.; Afanaseva, M. V.; Lagutina, M. A.; Kazanskii, K. S.: Comprehensive Characterization of Superabsorbent Polymer Hydrogels. Polym Bull 1990, 24, 107-113.

(2) Tanaka, T.; Fillmore, D.; Sun, S. T.; Nishio, I.; Swislow, G.; Shah, A.: PhaseTransitions in Ionic Gels. Phys Rev Lett 1980, 45, 1636-1639.

(3) Ohmine, I.; Tanaka, T.: Salt Effects on the Phase-Transition of Ionic Gels. Journal of Chemical Physics 1982, 77, 5725-5729.

(4) Hu, Y. H.; Chan, E. P.; Vlassak, J. J.; Suo, Z. G.: Poroelastic relaxation indentation of thin layers of gels. J Appl Phys 2011, 110 .

(5) Tanaka, T.; Nishio, I.; Sun, S. T.; Uenonishio, S.: Collapse of Gels in an ElectricField. Science 1982, 218, 467-469.

(6) Hirokawa, Y.; Tanaka, T.: Volume Phase-Transition in a Nonionic Gel. Journal of Chemical Physics 1984, 81, 6379-6380.

(7) Suzuki, A.; Tanaka, T.: Phase-Transition in Polymer Gels Induced by VisibleLight. Nature 1990, 346, 345-347.

(8) Beebe, D. J.; Moore, J. S.; Bauer, J. M.; Yu, Q.; Liu, R. H.; Devadoss, C.; Jo, B. H.: Functional hydrogel structures for autonomous flow control inside microfluidic channels. Nature 2000, 404, 588-+.

(9) Osada, Y.; Okuzaki, H.; Hori, H.: A Polymer Gel with Electrically Driven Motility. Nature 1992, 355, 242-244.

(10) Katchalsky, A.; Michaeli, I.: Polyelectrolyte Gels in Salt Solutions. Journal of Polymer Science 1955, 15, 69-86.

(11) Michaeli, I.; Katchalsky, A.: Potentiometric Titration of Polyelectrolyte Gels. Journal of Polymer Science 1957, 23, 683-696.

(12) Ricka, J.; Tanaka, T.: Swelling of Ionic Gels - Quantitative Performance of the Donnan Theory. Macromolecules 1984, 17, 2916-2921.

(13) Rubinstein, M.; Colby, R. H.; Dobrynin, A. V.; Joanny, J. F.: Elastic modulus and equilibrium swelling of polyelectrolyte gels. Macromolecules 1996, 29, 398-406.

(14) Dubrovskii, S. A.; Rakova, G. V.: Elastic and osmotic behavior and network imperfections of nonionic and weakly ionized acrylamide-based hydrogels. Macromolecules 1997, 30, 7478-7486.

(15) Dobrynin, A. V.; Colby, R. H.; Rubinstein, M.: Scaling Theory of Polyelectrolyte Solutions. Macromolecules 1995, 28, 1859-1871.

(16) Brannonpeppas, L.; Peppas, N. A.: Equilibrium Swelling Behavior of PhSensitive Hydrogels. Chem Eng Sci 1991, 46, 715-722.

(17) Marcombe, R.; Cai, S. Q.; Hong, W.; Zhao, X. H.; Lapusta, Y.; Suo, Z. G.: A theory of constrained swelling of a pH-sensitive hydrogel. Soft Matter 2010, 6, 784-793.

(18) Prudnikova, K.; Utz, M.: Electromechanical Equilibrium Properties of Poly(acrylic acid/acrylamide) Hydrogels. Macromolecules 2012, 45, 1041-1045.

(19) Hong, W.; Zhao, X. H.; Suo, Z. G.: Large deformation and electrochemistry of polyelectrolyte gels. J Mech Phys Solids 2010, 58, 558-577.

(20) Cai, S. Q.; Suo, Z. G.: Equations of state for ideal elastomeric gels. Epl-Europhys Lett 2012, 97. 
(21) Li, J. Y.; Hu, Y. H.; Vlassak, J. J.; Suo, Z. G.: Experimental determination of equations of state for ideal elastomeric gels. Soft Matter 2012, 8, 8121-8128.

(22) Hong, W.; Zhao, X. H.; Zhou, J. X.; Suo, Z. G.: A theory of coupled diffusion and large deformation in polymeric gels. J Mech Phys Solids 2008, 56, 1779-1793.

(23) Lifson, S.; Katchalsky, A.: The Electrostatic Free Energy of Polyelectrolyte Solutions .2. Fully Stretched Macromolecules. Journal of Polymer Science 1954, 13, 43-55.

(24) Prudnikova, K.; Utz, M.: Electromechanical Characterization of Polyelectrolyte Gels by Indentation. Macromolecules 2010, 43, 511-517.

(25) Day, J. C.; Robb, I. D.: Thermodynamic Parameters of Polyacrylamides in Water. Polymer 1981, 22, 1530-1533.

(26) Skouri, R.; Schosseler, F.; Munch, J. P.; Candau, S. J.: Swelling and Elastic Properties of Polyelectrolyte Gels. Macromolecules 1995, 28, 197-210.

(27) Arruda, E. M.; Boyce, M. C.: A 3-Dimensional Constitutive Model for the Large Stretch Behavior of Rubber Elastic-Materials. J Mech Phys Solids 1993, 41, 389-412.

(28) Boyce, M. C.; Arruda, E. M.: Constitutive models of rubber elasticity: A review. Rubber Chem Technol 2000, 73, 504-523.

(29) Gent, A. N.: A new constitutive relation for rubber. Rubber Chem Technol 1996, $69,59-61$.

(30) Horgan, C. O.; Saccomandi, G.: Constitutive models for compressible nonlinearly elastic materials with limiting chain extensibility. J Elasticity 2004, 77, 123-138.

(31) Lu, T. Q.; Huang, J. H.; Jordi, C.; Kovacs, G.; Huang, R.; Clarke, D. R.; Suo, Z. G.: Dielectric elastomer actuators under equal-biaxial forces, uniaxial forces, and uniaxial constraint of stiff fibers. Soft Matter 2012, 8, 6167-6173.

(32) Webber, R. E.; Creton, C.; Brown, H. R.; Gong, J. P.: Large strain hysteresis and mullins effect of tough double-network hydrogels. Macromolecules 2007, 40, 2919-2927. 